\title{
Laser Induced Formation of Buried Void Layer in Silicon
}

\author{
T. Sameshima*1, Y. Kanda ${ }^{* 1}$, M. Hasumi*1 ${ }^{1}$, T. Tatemichi*2 ${ }^{2}$, Y. Inouchi*2 ${ }^{2}$, and M. Naito*2 \\ ${ }^{* 1}$ Tokyo University of Agriculture and Technology, 2-24-16, Naka-cho, Koganei, Tokyo, 184-8588, Japan \\ Email: tsamesim@cc.tuat.ac.jp \\ $*^{2}$ Nissin Ion Equipment Co.,Ltd. Koka, Shiga 528-0068, Japan
}

\begin{abstract}
Buried void layer formation in silicon substrates was achieved by hydrogen ion implantation followed by $940-\mathrm{nm}$ infrared laser rapid heating. P-type silicon substrates coated with 100 -nm-thick thermally grown $\mathrm{SiO} 2$ layers were implanted with hydrogen atoms at $5 \times 10^{15}$ and $3 \times 10^{16} \mathrm{~cm}^{-2}$ at 60 $\mathrm{keV}$. Structural investigation with analysis of optical reflectivity spectra revealed that $3 \times 10^{16} \mathrm{~cm}^{-2}$ implantation caused a void layer with a void ratio of 0.04 at a depth of $250 \mathrm{~nm}$ close to the position of hydrogen peak concentration. Laser irradiation at $4.2 \times 10^{4} \mathrm{~W} / \mathrm{cm}^{2}$ increased the void ratio to 0.12 . No void layer was observed at ion implantation stage for sample with $5 \times 10^{15} \mathrm{~cm}^{-2}$-hydrogen atoms. Laser irradiation at $4.9 \times 10^{4} \mathrm{~W} / \mathrm{cm}^{2}$ formed a void layer with a void ratio of 0.013 at a depth of 255 nm.
\end{abstract}

DOI:10.2961/jlmn.2012.01.0018

Keywords: SOI, hydrogen implantation, laser heating, void layer, optical reflectivity

\section{Introduction}

Hydrogen ion implantation is an attractive method for fabrication of the silicon-on-insulator structure [1]. Highly dense hydrogen atoms are easily incorporated in the silicon surface regions by ion implantation method. Subsequent heating causes evaporation of hydrogen and formation of a buried void layer in silicon, which results in removing the top silicon layer from the underlying silicon substrate. Narrow and flat void layer is suitable for making removing surface flat and smooth.

In this paper, we propose millisecond-rapid laser heating to form buried void layer in hydrogen implanted silicon substrates. High intensity laser irradiation can rapidly heat the silicon surface region to a high temperature [2]. Laser rapid heating will easily evaporate hydrogen atoms implanted in silicon. Many silicon bonds will be broken associated with the hydrogen evaporation so that small voids will be formed in the silicon surface region. Laser rapid heating will simultaneously cure lattice damages caused by hydrogen ion implantation. We report analysis of optical reflectivity spectra of samples implanted with hydrogen atoms and subsequently heated by laser irradiation. The analysis confirms buried void layer formation. It also determines the depth of void layer and the density of voids. Moreover, the analysis reports change in crystalline volume ratio in the silicon surface region with laser irradiation. Via those analyses, we discuss the condition of laser-induced formation of bu-ried void layer.

\section{Experimental Details}

P-type silicon substrates with a resistivity of $20 \Omega \mathrm{cm}$ and a thickness of $520 \mu \mathrm{m}$ were prepared. The both surfaces were coated with 100 -nm-thick thermally grown $\mathrm{SiO}_{2}$ layers. Hydrogen $2 \mathrm{H}^{+}$ions were implanted to the top surface of silicon substrates at $60 \mathrm{keV}$, with doses of $5 \times 10^{15}$ and $3 \times 10^{16} \mathrm{~cm}^{-2}$. The in-depth profile of hydrogen atoms were estimated by numerical calculation program, as shown in Fig. 1. Hydrogen atoms had a peak concentration at $230 \mathrm{~nm}$ deep from the silicon surface.
Samples were irradiated with infrared semiconductor laser. Figure 2 shows a schematic apparatus of laser heating A 940-nm infrared semiconductor laser beam with a maximum power of $23 \mathrm{~W}$ was introduced by an optical fiber.

The optical fiber and optics with lens were mounted on a $\mathrm{X}-\mathrm{Y}$ mobile stage. The laser was moved in the Y-direction. It was also moved in the X-direction with a step of $100 \mu \mathrm{m}$. The laser beam was focused by lens to a spot with a Gauss-

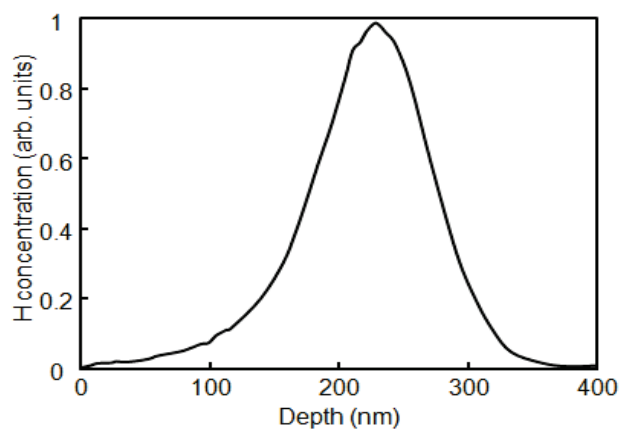

Fig. 1: In-depth concentration of hydrogen atoms calculated by TRIM.

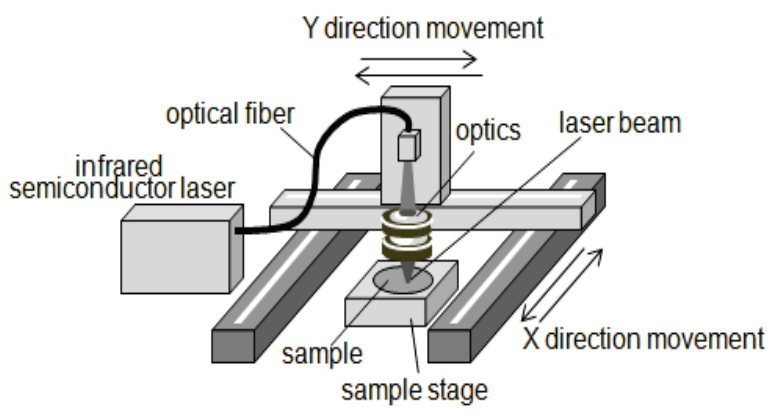

Fig. 2: Schematic apparatus of infrared semiconductor laser heating. 
ian intensity distribution with a diameter of $200 \mu \mathrm{m}$ at full width at half maximum at the sample surface. Samples were placed on a stage at a normal direction to the laser beam. The samples were irradiated with the laser beam at power densities ranging from $4.0 \times 10^{4}$ to $4.9 \times 10^{4} \mathrm{~W} / \mathrm{cm}^{2}$ for $4 \mathrm{~ms}$ conducted by moving the laser beam at $5 \mathrm{~cm} / \mathrm{s}$. A numerical heat flow simulation estimated that the silicon surface was heated to $780^{\circ} \mathrm{C}$ at maximum by laser heating at $4.9 \times 10^{4} \mathrm{~W} / \mathrm{cm}^{2}$ for $520 \mu \mathrm{m}$-thick samples.

Optical reflectivity spectra were measured between 250 and $1100 \mathrm{~nm}$ using a conventional spectrometer. They were analyzed using a numerical calculation program, which was constructed with the optical interference effect with multiple-layered structures with top silicon layer and underlying void layers, as shown in Fig. $3[3,4]$. The optical reflectivity depends on the complex refractive indexes of those layers. Using the effective-medium approximation model, we introduced the complex refractive indexes, $\mathrm{n}_{\alpha}$ for the top silicon layer as partially amorphized layer with a crystalline volume ratio, $\alpha$, and $\mathrm{n}_{\beta}$ for the void layer as partially void layers with a void ratio $\beta$, given as

$$
\begin{aligned}
& n_{\alpha}=\alpha n_{\mathrm{c}}+(1-\alpha) n_{\mathrm{a}} \\
& n_{\beta}=\beta n_{\mathrm{air}}+(1-\beta) \mathrm{n}_{\alpha}
\end{aligned}
$$

where $n_{\mathrm{c}}, n_{\mathrm{a}}$, and $n_{\text {air }}$ are complex refractive indexes of crystalline silicon, amorphous silicon, and $\operatorname{air}(=1)$. The values of the parameters of thickness of each layer, $\alpha$, and $\beta$ were changed for each layer to calculate the reflectivity. Difference in reflectivity spectra was introduced by subtracting spectrum of samples from the spectra of single crystalline silicon to precisely analyze structure of surface regions.

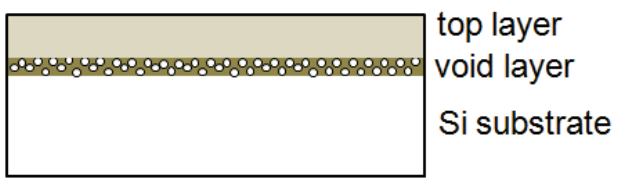

Fig. 3: Schematic structure of silicon surface region caused by hydrogen ion implantation and laser heating.

Figure 4 shows calculated optical reflectivity spectra of 255-nm-thick partially amorphized layer with $\alpha$ of 0.8 at the top surface, 10 -nm-thick partially void layer with $\beta$ of 0.2 underlying 255 -nm-thick perfect crystal-line top layer, and single crystalline silicon (a), and differences in those optical reflectivity spectra, which were obtained by subtracting optical reflectivity spectra of the layered structures by the optical reflectivity spectrum of single crystalline silicon (b). The $E_{1}$ and $E_{2}$ peaks around 280 and $370 \mathrm{~nm}$ appeared for every case in Fig. 4(a). In the case of the partially amorphized top layer, those peak heights were slight ly lower than that of single crystalline because those peaks sensitively reflect the density of states of crystalline silicon. The optical reflectivity spectra of calculated samples with the partially amorphized top layer and partially void layer had small fringes from 400 to $1100 \mathrm{~nm}$. Those fringes resulted from the optical interference effect caused by the different in complex refractive indexes between the top partially amorphized layer and crystalline silicon, or the top crystalline layer and buried void layer. The fringes appeared in the positive region in the difference spectra for sample with the partially amorphized top layer, as shown in Fig. 4(b) because amorphous silicon has higher refractive indexes that those of crystalline silicon between 400 and $1100 \mathrm{~nm}$. On the other hand, the fringes oscillated almost symmetrically on the zero point in the case of the buried void layer, as shown in Fig. 4(b). The results of Fig. 4 indicate that analysis of optical reflectivity spectra in ultraviolet, visible, and infrared regions provide information on the void layer and crystalline volume ratio near surface regions. The most possible in-depth distributions of the void and crystalline volume ratio were obtained by fitting calculated spectra to experimental ones.

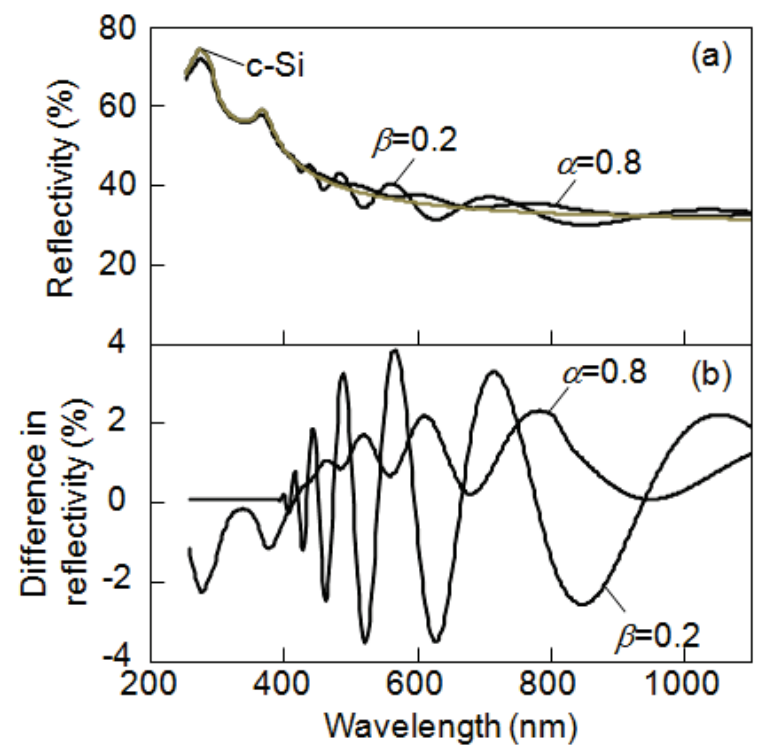

Fig. 4: Calculated optical reflectivity spectra (a) and differences in those reflectivity spectra (b) for the cases of 255-nm-thick partially amorphized top layer with $\alpha$ of $0.8,10$-nm-thick partially void layer with $\beta$ of 0.2 at a depth of $255 \mathrm{~nm}$ as well as single crystalline silicon shown in (a).

\section{Results and discussions}

Solid curves in Fig. 5 show differences in experimental reflectivity spectra of samples as- $5 \times 10^{15}-\mathrm{cm}^{-2}$-hydrogen-ion implanted (a), laser annealed at $4.2 \times 10^{4} \mathrm{~W} / \mathrm{cm}^{2}$ (b), and $4.9 \times 10^{4} \mathrm{~W} / \mathrm{cm}^{2}$ (c). Dashed curves in Fig. 5 show calculated differences in reflectivity spectra conducted using our theory described above. The as-ion-implanted sample shows small fringes between 400 and $1100 \mathrm{~nm}$. They appeared in the positive region, as shown in Fig. 5(a). It means that the surface region was partially amorphized by hydrogen ion implantation. Laser heating at $4.2 \times 10^{4}$ $\mathrm{W} / \mathrm{cm}^{2}$ increased the amplitude of fringes and moved them 
to zero level, as shown in Fig. 5(b). It means that a void layer was formed in silicon substrate by the laser heating. Laser heating at $4.9 \times 104 \mathrm{~W} / \mathrm{cm} 2$ further increased the amplitude of fringes. The fringes oscillated almost symmetri-

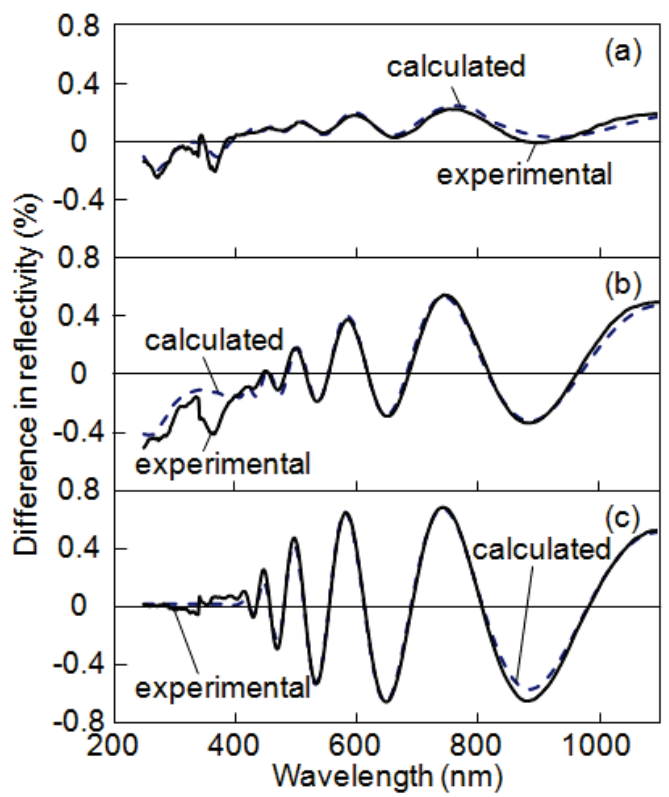

Fig. 5: Differences in experimental reflectivity spectra of samples as- $5 \times 10^{15}-\mathrm{cm}^{-2}$-hydrogen-ion implanted (a), laser annealed at $4.2 \times 10^{4} \mathrm{~W} / \mathrm{cm}^{2}$ (b), and $4.9 \times 10^{4}$ $\mathrm{W} / \mathrm{cm}^{2}$ (c). Dashed curves show calculated differences in reflectivity spectra.

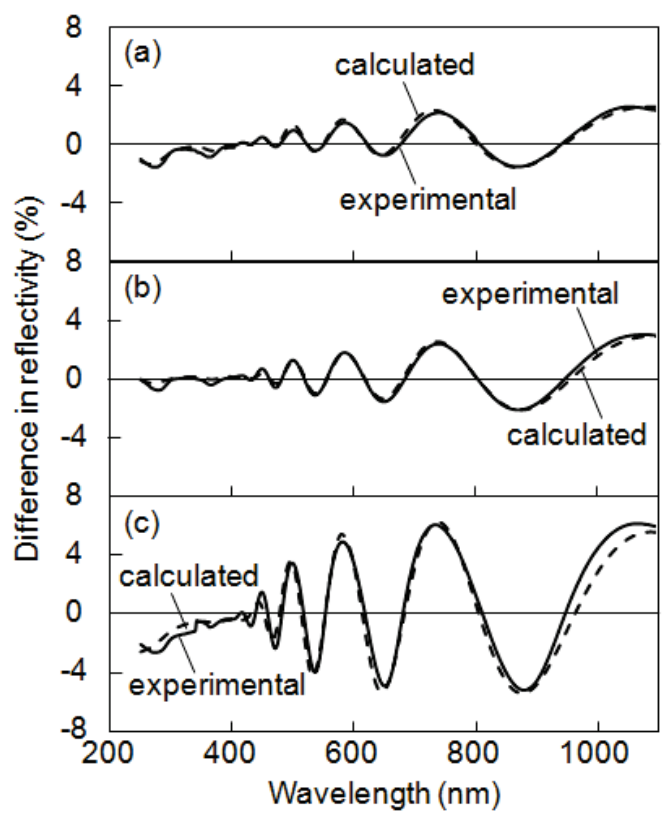

Fig. 6: Differences in experimental reflectivity spectra of samples as- $3 \times 10^{16}-\mathrm{cm}^{-2}$-hydrogen-ion implanted (a), laser annealed at $4.0 \times 10^{4} \mathrm{~W} / \mathrm{cm}^{2}$ (b), and $4.2 \times 10^{4}$ $\mathrm{W} / \mathrm{cm}^{2}$ (c). Dashed curves show calculated differences in reflectivity spectra. cally on the zero point as shown in as shown in Fig. 5(c). On the other hand, the phase of oscillation of the fringes was the same between Fig. 5(b) and 5(c). Those mean that the top surface region maintained the same thickness, and that the void ratio $\beta$ was increased by the laser heating at $4.9 \times 10^{4} \mathrm{~W} / \mathrm{cm}^{2}$.

Solid curves in Fig. 6 show differences in experimental

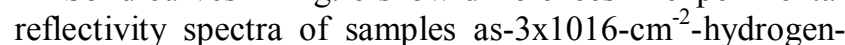
ion implanted (a), laser annealed at $4.0 \times 10^{4} \mathrm{~W} / \mathrm{cm}^{2}(\mathrm{~b})$, and $4.2 \times 10^{4} \mathrm{~W} / \mathrm{cm}^{2}$ (c). Dashed curves in Fig. 6 show calculated differences in reflectivity spectra. The as-ion-implanted sample shows fringes between 400 and $1100 \mathrm{~nm}$ with rather large amplitudes compared with the samples implanted with $5 \times 10^{15}-\mathrm{cm}^{2}$-hydrogen ions, as shown in Figs. 6(a) and 5(a). The oscillation central point was close to the zero point, as shown in Fig. 6(a). It means that $3 \times 10^{16}-\mathrm{cm}^{-2}-$ hydrogen-ion implantation formed buried void layer and partially amorphized the top surface region si-multaneously. Some hydrogen atoms probably recombined and made hydrogen molecules to increase gas pressure in silicon during their implantation. Hydrogen gases broke silicon bonds. Laser heating at $4.2 \times 10^{4} \mathrm{~W} / \mathrm{cm}^{2}$ increased the amplitude of fringes, as shown in Fig. 6(c). It means that the void ratio $\beta$ was increased by laser heating. The phase of oscillation of the fringes was almost the same among Figs. 6(a) to 6(c). It indicates that the void layer maintained the same depth during implantation and laser heating.

Best fitting calculated spectra to the experimental spectra shown in Figs. 5 and 6 gave information of sample structures. Figure 7 shows summary of analysis of $\alpha$ (a), the thickness at the top layer (b), the thickness of the void layer (c), and $\beta(\mathrm{d})$ as functions of the laser intensity. In-

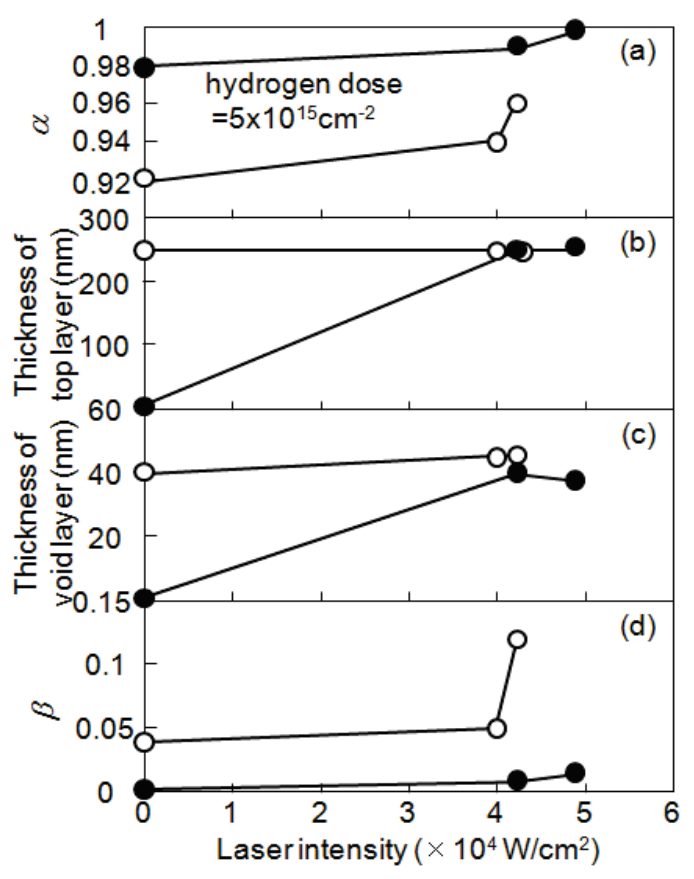

Fig. 7: $\alpha(\mathrm{a})$, thickness at the top layer (b), thickness of the void layer (c), and $\beta(\mathrm{d})$ as functions of the laser intensity for samples implanted with hydrogen at $5 \times 10^{15}$ (solid marks) and $3 \times 10^{16} \mathrm{~cm}^{-2}$ (open marks). 
crease in $\alpha$ shown in Fig. 7(a) means that the top silicon layer was recrystalllized well by laser rapid heating. The void layers were formed 255 and $250 \mathrm{~nm}$ from the surface for samples implanted with $5 \times 10^{15}$ and $3 \times 10^{16} \mathrm{~cm}^{-2}$ hydrogen atoms respectively, as shown in Fig. 7(b). The depths were just close to the peak concentration of hydrogen atoms implanted at $60 \mathrm{keV}$, as shown in Fig. 1. The void layers had a thickness of about $40 \mathrm{~nm}$, as shown in Fig. 7(c). Small voids probably distributed in the regions. $\beta$ was increased to 0.12 by laser heating at $4.2 \times 10^{4} \mathrm{~W} / \mathrm{cm}^{2}$ for samples implanted with $3 \times 10^{16}-\mathrm{cm}^{-2}$-hydrogen atoms, although a void layer with $\beta$ of 0.04 was already formed at implantation, as shown in Fig. 7(d). $\beta$ was very low of 0.013 in the case of high power laser heating at $4.9 \times 10^{4} \mathrm{~W} / \mathrm{cm}^{2}$ for samples implanted with $5 \times 10^{15}-\mathrm{cm}^{-2}$-hydrogen atoms. The low hydrogen concentration was not effective to form void layer. The sample maintained a smooth surface during all of process steps. Further optimization will achieve narrow and flat void layer with high void ratio.

\section{Summary}

We reported buried void layer formation by laser rapid heating of hydrogen implanted silicon substrates. P-type silicon substrates coated with $100 \mathrm{~nm}$ thick thermally grown $\mathrm{SiO}_{2}$ layers were implanted with hydrogen atoms at $5 \times 10^{15}$ and $3 \times 10^{16} \mathrm{~cm}^{-2}$ at $60 \mathrm{keV}$. An apparatus of $940-\mathrm{nm}$ infrared semiconductor laser irradiation was constructed with an optics focusing the laser beam and a mechanical stage moving laser beam. Structural investigation was conducted by analysis of optical reflectivity spectra with a nu- merical calculation program constructed with the optical interference effect with multiple-layered structures. $3 \times 10^{16}$ cm-2-hydrogen implantation caused a void layer with a void ratio of 0.04 at a depth of $250 \mathrm{~nm}$ close to the position of hydrogen peak concentration. Laser irradiation at $4.2 \times 10^{4} \mathrm{~W} / \mathrm{cm}^{2}$ increased the void ratio to 0.12 . On the other hand, no void layer was observed at ion implantation stage for sample with $5 \times 10^{15} \mathrm{~cm}^{-2}$-hydrogen atoms. Laser irradiation at $4.9 \times 10^{4} \mathrm{~W} / \mathrm{cm}^{2}$ formed a void layer with a void ratio of 0.013 at a depth of $255 \mathrm{~nm}$..

\section{Acknowledgments}

This work was partly supported by Grant-in-Aid for Science Research C (No. 22560292) from the Ministry of Education, Culture, Sports, Science and Technology of Japan, and Takahashi Industrial and Economic Research Foundation.

\section{References}

[1] S. Personnic, A. Tauzin, K. Bourdelle, F. Letertre, N. Kerneves, F. Laugier, N. Cherkashin, A. Claverie and R. Fortunier, IIT2006 AIP conference proceeedings (2006) pp.65-68

[2] K. Ukawa, Y. Kanda, T. Sameshima, N. Sano, M. Naito and N. Hamamoto: Jpn. J.Appl. Phys. 49 (2010) 076503.

[3] M. Born and E. Wolf: Principles of Optics (Pergamon, New York, 1974) Chaps. 1 and 13.

[4] T. Sameshima, Y. Matsuda, Y.Andoh, and N. Sano, Jpn. J.Appl. Phys. 47 (2008) 1871.

(Received: June 3, 2011, Accepted: January 16, 2012) 